\section{Coupling mechanism between geoacoustic emission and elec- tromagnetic anomalies prior to earthquakes}

\author{
Viacheslav Pilipenko, Evgeniy Fedorov \\ Institute of the Physics of the Earth, \\ Moscow, Russia
}

\begin{abstract}
Micro-cracking in the earthquake preparation zone is accompanied by the generation of acoustic emission (AE). Even low-intensity $\mathrm{AE}$ can essentially modify the underground fluid dynamics owing to the influence of high-frequency acoustic field on filtration process. Laboratory experiments show that acoustic impact on pour sample destroys a film with bounded water and results in a steep increase of its permeability up to 2 orders of magnitude. Impulsive acoustic fields also decrease the effective viscosity of the fluid. The occurrence in the crust under pressure of a region with distinct hydrodynamic and electrokinetic parameters will result in an appearance of anomalous telluric and magnetic fields on the surface above. This effect is estimated analytically using a simple model with an ellipticshaped inhomogeneity. The suggested hypothesis about possible coupling between $\mathrm{AE}$ and geoelectrical anomalies needs observational verification.
\end{abstract}

\section{Introduction}

Dynamics of the crust fluid plays a key role in seismic process and volcanic activity. Fluids and particularly high fluid pressure (such as large over-pressured volumes of rock adjoining fault zone and narrow fault itself) are considered to play a major role in determining the long-term fault strength and the character of its rapture. ${ }^{1}$ The fluid dynamics is very sensitive to the crust stress, so its ground monitoring with the use of electromagnetic methods may provide a tool for the earthquake (EQ). ${ }^{2-5}$ Fluid flow can be monitored with observations of water levels in wells ${ }^{6}$ and with detection of ultra-low-frequency (ULF) electromagnetic fields arising due to electrokinetic effect. ${ }^{7-10}$

The most intense variations of the underground water level are observed from several days to months before $\mathrm{EQ}$, and these variations can be detected at distances up to $500 \mathrm{~km}$ from epicenter and reach amplitudes up to few meters. Probably, the crust volume deforma- tions, which can reach values $\simeq 10^{-6}$, can produce variations of water levels of the same order, that is $\Delta h / h \simeq \varepsilon$, which is much less than the observed values. That is why another mechanism was suggested by Makarenko and Groza $^{11}$ to explain the underground water level variations related to seismicity. During microcracking a generation of acoustic emission (AE) in the frequency range from ultra- to infra-sound (ELF) takes place, a wavelength being about the crack scale. ${ }^{12-14}$ The source of $\mathrm{AE}$ could be an entire region of the $\mathrm{EQ}$ preparation. There are numerous experimental evidences $^{15}$ indicating that the EQ preparation region is filled with acoustic fields having a noticeable intensity. For example, Morgunov et $a l .{ }^{16}$ recorded distinct anomaly in $800-1200 \mathrm{~Hz}$ AE behavior $\sim 16 \mathrm{~h}$ before the $M=7.0$ Spitak EQ at the epicentral distance of $80 \mathrm{~km}$.

Even low-intensity AE can essentially modify the underground fluid dynamics owing to the influence of high-frequency acoustic field on filtration process of the crust fluid, as was demonstrated by Chersky et al., ${ }^{17}$ and Zarev et $a l .{ }^{18}$ Velocity $\mathbf{u}$ of filtration of a fluid with the dynamic viscosity $\eta$ under the influence of the fluid pressure gradient $\nabla P$ is described by Darcy's law (Eq.1):

$$
\mathbf{u}=-\left(\frac{k}{\eta}\right) \nabla P
$$

The permeability $k$ is determined by the porosity $m$ and the pour diameter $d$ as follows (Eq.2):

$$
k \propto d^{2} \frac{m^{3}}{(1-m)^{2}}
$$

The actual diameter $d$ is less than the pour scale owing to occurrence of double electric layer with bounded water in it.

Laboratory experiments showed that acoustic impact (especially an impulsive one) on porous sample results in a steep increase of its permeability up to 2 orders of magnitude. ${ }^{17,18}$ Acoustic wave destroys a film with a bounded water, which results in an increase of the effective porosity $m$. Additionally, even the capillars that were not involved into filtration, do so after the AE impact. Acoustic field also decrease the effective viscosity of the fluid $\eta$. In summary, the filtration coefficient $K \alpha \mathrm{k} / \eta$ grows and increases the Darcy's velocity $\mathbf{u}$.

\section{Electrokinetic anomaly above a crust with heterogeneous parameters}

Significance of AE for the formation of EQ precursors is not restricted by hydrogeodynam-
Correspondence: Viacheslav A. Pilipenko, Institute of the Physics of the Earth, B. Gruzinskaya 10, Moscow 123995, Russia.

E-mail: space.soliton@gmail.com

Key words: earthquake prediction, acoustic emission, electromagnetic methods, electrotelluric field, crust fluid.

Acknowledgements: this study is supported by the RFBR grant 13-05-12091. The useful comments from K. Shiokawa are appreciated.

Received for publication: 24 July 2013 .

Revision received: 30 May 2014.

Accepted for publication: 4 July 2014.

This work is licensed under a Creative Commons Attribution NonCommercial 3.0 License (CC BYNC 3.0).

(C) Copyright V. Pilipenko and E. Fedorov, 2014 Licensee PAGEPress, Italy

Research in Geophysics 2014; 4:5008

doi:10.4081/rg.2014.5008

ic phenomena only. Mechanism of modification of the crust parameters by $\mathrm{AE}$ can be applied to electrokinetic phenomena. The relation between the current density and the gradients of pressure $P$ and electric potential $\varphi$ is as follows (Eq.3): ${ }^{19}$

$$
\mathbf{j}=-\sigma(\nabla \varphi+C \nabla P)
$$

Here $\sigma$ is the conductivity, $C=\varepsilon £ / \sigma \eta$ is the streaming potential, $\zeta$ is the zeta-potential, and $\varepsilon$ is the dielectric permeability. Change of viscosity $\eta$ results in the change of streaming potential $C$. Thus, the impact of AE causes the occurrence of a region in the crust with modified hydrogeodynamic and electrokinetic parameters, and subsequently produces anomalous telluric and magnetic fields.

The electromagnetic field produced by electrokinetic process can be, in principle, calculated according to the following scheme. Using the condition $\nabla \times \mathbf{j}=0$, the equation for the potential $\varphi$ is obtained (Eq.4):

$$
\nabla(\sigma \nabla \varphi)=-\nabla(\sigma C \nabla P)
$$

From the above equation the distribution of potential $\varphi$ and electric field, $\mathbf{E}=-\nabla \varphi$, can be found. Finally, the magnetic field $\mathbf{B}$ can be derived through the current $\mathbf{j}$ from the solution of the magnetostatic problem (Eq.5):

$$
[\nabla \times \mathbf{B}]=\mu_{0} \mathbf{j}, \quad \nabla \cdot \mathbf{B}=0
$$

Numerical estimates of electric and magnetic fields produced by electrokinetic current in a layered model were made by Fitterman. ${ }^{20} \mathrm{He}$ 
showed that the problem of electromagnetic fields during the fluid flow through a patch can be reduced to the problem of fields from a grounded dipole. Another model of electrokinetic source was suggested by Fenoglio et al. ${ }^{21}$ This numerical model assumed as a possible mechanism of generation of oscillatory electromagnetic signals in ULF band the stop-andstart fracture propagation. Rupture of seals in response to a tectonic loading would initiate shear fractures that propagate into the lowpressure compartment, enabling the rapid movement of fluid from the high-pressure compartment. This process was modeled with an electrokinetic current flow along a thin vertical layer (fault) located at some depth below the Earth's surface.

In contrast with previous numerical models, here we develop a simplified, but analytical model, which provides straightforward expressions for the expected electromagnetic effect above a region with modified electrokinetic parameters. Originally, this model has been suggested by Fedorov et al. ${ }^{22}$ for the interpretation of ULF magnetic disturbances produced by irregular flow of fluid through the crust inhomogeneity.

\section{Model of underground ellipti- cal inhomogeneity}

Here we consider electromagnetic fields which are generated by a flow of the crust fluid through a region with a modified electrokinetic potential $C$. Electromagnetic fields can be calculated in a quasi-static approximation, which is valid in the ULF frequency range and distances about the epicenter depths. Hence, all the temporal variations of generated elec- tromagnetic fields are totally determined by temporal variations of a fluid flow. An irregular character of the fluid flow results in a fluctuating anomalous electromagnetic field. In most geophysical applications, a source region is small as compared to the distance from a source to an observational site on the ground. Therefore, it is sufficient to consider the point current source with an effective moment $\mathbf{M}$.

We use the rectangular coordinate system $(x, y, z)$ with axis $z$ downward, and plane $z=0$ corresponding to the ground surface. The source region is imagined as an ellipsoid with the main axes $a, b, c$ (parallel to the coordinate axes $x, y, z$, correspondingly) with constant conductivity and streaming potential $C_{i}$ (Figure 1). The advantage of such ellipsoid model is that under a proper choice of $a, b$, and $c$ scales, this model reduces to all known models of electrokinetic source. Outside the ellipsoid, the medium conductivity $\sigma_{e}$ and the streaming potential $C_{e}$ are different, but constant. The electromagnetic field is excited by the current, driven by the crust pressure gradient $\nabla P$, directed along $x$ axis, and producing the pressure drop $\Delta P$ across the distance $a$.

We introduce the potentials $\psi_{i}=\varphi_{i}+C_{e} P$ and $\psi_{e}=\varphi_{e}+C_{e} P$, where $\varphi_{e}$ and $\varphi_{i}$ are electrostatic potentials outside and inside the ellipsoid. The net electric current $\mathbf{j}$ is a sum of the driver current $\mathbf{j}_{\mathrm{i}}$ and back conductivity current $\mathbf{j}_{\mathrm{b}}$. The driver current is $\mathbf{j}_{i}=-\sigma_{i} \Delta C \nabla P$ inside the ellipsoid and $\mathbf{j}_{\mathrm{i}}=0$ outside it, where $\Delta C=C_{i}-C_{e}$ is the contrast of streaming potentials inside and outside the ellipsoid. For the potential $\psi$ the equation $\nabla^{2} \psi=0$ is valid within and outside ellipsoid. Besides that, the potential $\psi$ and the total current normal to the boundary are to be continuous at the ellipsoid surface $S$, that is $\left.\left\{\psi_{e}-\psi_{i}\right\}\right|_{s}=0$, and $\left.\left\{\sigma_{e}(\mathbf{n} \nabla) \psi_{e}-\sigma_{i}(\mathbf{n} \nabla) \psi_{i}\right\}\right|_{s}=-\left.\sigma_{i} \Delta C(\mathbf{n} \nabla) P\right|_{s}$. At the interface air-ground the vertical electric current must vanish, that is $\left.\partial_{z} \psi\right|_{z=0}=0$.

When the center of the ellipsoid is at the depth $h>>\max (a, b, c)$ and only disturbances far from the ellipsoid are considered, the influence of the ground-atmosphere boundary on the polarization of such conductive ellipsoid can be neglected. The problem of the influence of spreading back currents on the magnetic effects of electrokinetic source is mathematically similar to the problem of the polarization of a dielectric ellipsoid immersed in homogeneous electric field.23 Based on this analogy, we have obtained that for the estimate of electromagnetic field far from a source, the ellipsoid with current $\mathbf{j}_{\mathrm{i}}$ can be replaced with the point current source with the effective moment (Eq.6):

$$
\mathbf{M}=\frac{\mathbf{M}_{i}}{1-n+p}
$$

where $\mathbf{M}_{\mathrm{i}}$ is the moment of the electrokinetic currents inside the ellipsoid (Eq.7):

$$
\begin{aligned}
& \mathbf{M}_{i}=\int \mathbf{j}_{i} d V=-(4 \pi / 3) a b c \sigma_{i} \Delta C \nabla P \\
& =-(4 \pi / 3) b c \sigma_{i} \Delta C \Delta P \hat{\mathbf{e}}_{x}
\end{aligned}
$$

In (Eq.6) the factor $p=\left(\sigma / \sigma_{e}\right) n$ is derived through the geometric factor of depolarization $n$. For an arbitrary ellipsoid the factor $n$ is derived via the elliptic functions, and only for a rotational ellipsoid this factor is derived via elementary functions. For the elongated along $z$ ellipsoid, when $c>>\max (a, b)$, this factor is $n \simeq b /(a+b)$. For a thin current sheet, i.e. when additionally $a>>b$, one obtains $n \simeq b / a<<1$.

The relation between the imposed current $\mathbf{j}_{\mathrm{i}}$

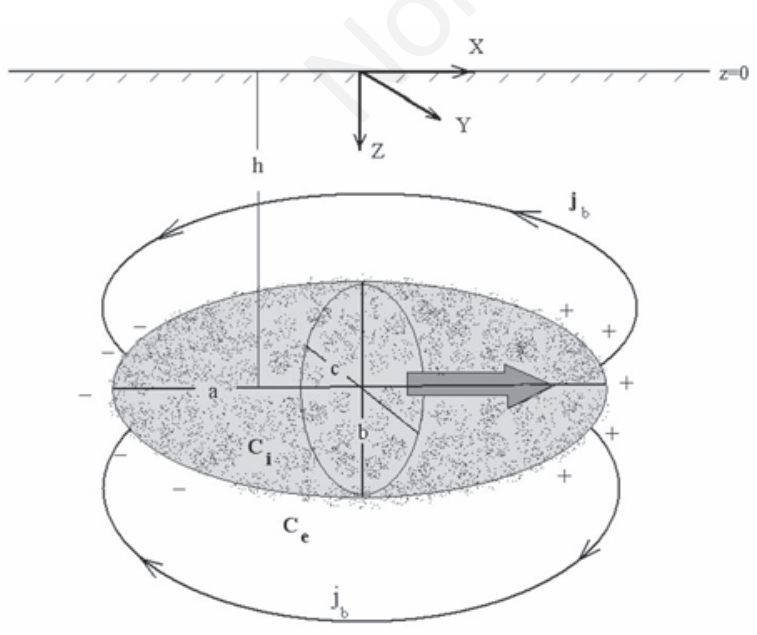

Figure 1. A sketch illustrating the ellipsoid model of the region with electrokinetic parameters modified by enhanced acoustic emission. Large arrow indicates a direction of the fluid pressure gradient.

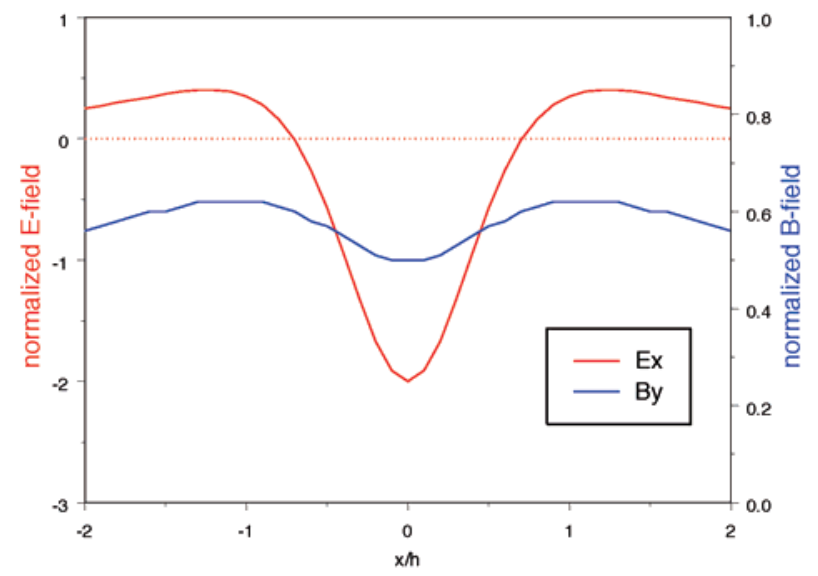

Figure 2. Electromagnetic anomaly above the region with enhanced acoustic emission: the profile along $\nabla P$ at $y=0$. 
and the total current $\mathbf{j}$ inside the ellipsoid, including the back current $\mathbf{j}_{\mathrm{b}}$, is as follows (Eq.8):

$$
\mathbf{j}=-\frac{1-n}{1-n+p} \mathbf{j}_{i}
$$

It can be seen from (Eq.8) that the role of the back current is controlled by the contrast of conductivities $\sigma_{i} / \sigma_{e}$ and the geometrical factor $n$.

In the case, when the conductivity in the generation region is much higher than outside, $\sigma_{i} \gg>\sigma_{e}$, and $p=\left(\sigma / \sigma_{e}\right) n>>1$, the back conductivity current nearly totally compensates the electrokinetic current, i.e. $\mathbf{j}_{b} \simeq-\mathbf{j}_{i}$. Hence, the total current is small, i.e. $\mathbf{j} \ll \mathbf{j}_{i}$. The total current and current moment both drop $p^{(x)}$ times, as follows (Eq.9):

$$
\mathbf{j} \simeq \mathbf{j}_{i} / p \quad \mathbf{M} \simeq \mathbf{M}_{i} / p
$$

Further, we consider the case when the contrast between the conductivities inside and outside ellipsoid is small, $\sigma_{i} \simeq \sigma_{e}$, and factor $p<<1$. In this case, the influence of the back current on the effective current moment can be neglected, i.e. (Eq.10):

$$
\mathbf{j} \simeq \mathbf{j}_{i}, \quad \mathbf{M} \simeq \mathbf{M}_{i}
$$

\section{Electromagnetic fields on the ground}

When an observational site is far away from the epicenter, the influence of the groundatmosphere interface can be neglected. In homogeneous space, in the dipole approximation the electrostatic potential $\varphi$ and magnetic field $\mathbf{B}$ produced by the current moment $\mathbf{M}=(M, 0,0)$ situated at depth $(0,0, h)$ are as follows (Eq.11):

$$
\varphi=\frac{\mathbf{M} \cdot \mathbf{R}_{1}}{4 \pi \sigma_{e} R_{1}^{3}} \mathbf{B}=\frac{\mu_{o}}{4 \pi} \frac{\mathbf{M} \times \mathbf{R}_{1}}{R_{1}^{3}}
$$

where

$$
R_{1}=\sqrt{x^{2}+y^{2}+(z-h)^{2}}
$$

When the observational site is at distance from epicenter comparable to the source depth, the influence of ground-atmosphere interface should be taken into account. The potential $\varphi$, which satisfies the boundary condition at the interface, can be found with the mirror images method from the elementary solution (Eq.11), obtained for an infinite homogeneous space. The electric field components at $z \geq 0$ are as follows (Eq.12):

$$
\begin{aligned}
& E_{x}=\frac{M}{4 \pi \sigma_{e}}\left[\left(2 x^{2}-y^{2}\right)\left(\frac{1}{R_{1}^{5}}+\frac{1}{R_{2}^{5}}\right)-\frac{(z-h)^{2}}{R_{1}^{5}}-\frac{(z+h)^{2}}{R_{2}^{5}}\right] \\
& E_{y}=\frac{M}{4 \pi \sigma_{e}}\left[3 x y\left(\frac{1}{R_{1}^{5}}+\frac{1}{R_{2}^{5}}\right)\right] \\
& E_{z}=\frac{M}{4 \pi \sigma_{e}} 3 x\left[\frac{(z-h)}{R_{1}^{5}}+\frac{(z+h)}{R_{2}^{5}}\right]
\end{aligned}
$$

where

$$
R_{2}=\sqrt{x^{2}+y^{2}+(z+h)^{2}}
$$

Magnetic field from the current system (Eq.12) can be found by straightforward and lengthy calculations. As a result we obtain (Eq.13):

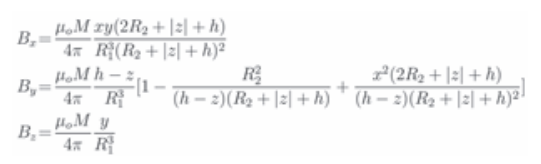

Formally, the case without ground-atmosphere boundary can be obtained directly from these relationships by putting $R_{2}^{-1} \rightarrow 0$ for $\mathrm{E}$ field (Eq.12) and $\left(R_{2}+|z|+h\right)^{-1} \quad 0$ for B field (Eq.13).

\section{Spatial and polarization struc- ture of electromagnetic anomaly}

For illustration of basic features of anomalous electromagnetic field on the Earth surface $(z=0)$ above the region with modified electrokinetic parameters the spatial field variations are analyzed. The electric and magnetic components can be presented in the form (Eq.14):

$$
B=\frac{\mu_{o} M}{4 \pi h^{2}} \bar{B}(\bar{x}, \bar{y}) E=\frac{M}{4 \pi \sigma_{e} h^{3}} \bar{E}(\bar{x}, \bar{y})(14)
$$

where $\overline{\mathrm{E}}$ and $\overline{\mathrm{B}}$ are dimensionless functions of normalized distances $\overline{\mathrm{x}}=x / h$, and $=\overline{\mathrm{y}}=y / h$.

The functions $\overline{\mathrm{E}}(\overline{\mathrm{x}}, \overline{\mathrm{y}})$ and $\mathrm{B}(\overline{\mathrm{x}}, \overline{\mathrm{y}})$, describing the spatial field structure, have been plotted along the direction of electrokinetic current $(y=0)$ (Figure 2) and across it $(x=0)$ (Figure 3). The expressions (Eqs.12,13) show that the presence of the air-ground boundary noticeably modifies electromagnetic fields produced by an underground source. The peak values of $\mathrm{E}_{x}$ and $\mathrm{B}_{y}$ above a source differ by nearly 2 times.
The ratio between electric and magnetic components (i.e. apparent impedance $Z_{x y}=$ $\left.\mu_{0} E_{\chi} / B_{y}\right)$ of disturbance varies with distance from a source. Along the profile co-located with $\nabla P$ (Figure 4, left panel), just above a source this value is high $Z_{x y}>>1$, but away from the source it decreases to low values, $Z_{x y}<<1$. Spatial changes in the value of an apparent impedance are related to different decay rate with distance from a source of electric, $E \alpha R^{-3}$, and magnetic, $B \alpha R^{-2}$, components.

The ratio between vertical and horizontal magnetic components (Figure 4, right panel) also varies from very small values near the source, $B / B_{y}<<1$, to relatively large values, $B_{L} / B_{y}>>1$. Thus the criteria of the increased values of $B / B_{y}$ often used for discrimination of underground sources from atmosphere/ionosphere sources cannot be considered as univer$\mathrm{sal}^{24}$

It is hard to expect that an observer will ever be so lucky to make measurements just above a seismic source. Realistically, all existing and future observations are away from the epicenter. Therefore, one should expect the electromagnetic fields produced by an underground electrokinetic source must have $Z<<1$ and $B / B>1$. These features may help to discriminate anomalous signals from underground sources from the typical magnetospheric activity, which commonly have $Z<<1$ and $B_{Z} / B$ $<<1 .{ }^{25}$

The magnitude of electromagnetic effect on the ground, without account for geometrical factors can be estimated with relationships (Eq.15):

$$
\frac{E}{\bar{E}}=\frac{b c}{3 h^{3}}\left(\frac{\sigma_{i}}{\sigma_{e}}\right) \Delta C \Delta P \frac{B}{\bar{B}}=\frac{\mu_{0} b c \sigma_{i}}{3 h^{3}} \Delta C \Delta P
$$

The typical value of the apparent impedance $Z$ of electromagnetic disturbance observed above an electrokinetic source, as follows from (Eq.16) is to be:

$$
\frac{Z}{\bar{Z}}=\frac{\mu_{o} E}{B}=\left(\sigma_{e} h\right)^{-1}
$$

Thus, this impedance is determined by the depth-integrated crust conductivity $\Sigma_{e}=\sigma_{e} h$.

To estimate the expected effect we assume that an anomaly is situated at $h=8 \mathrm{~km}$ with the transverse scales $b=c=4 \mathrm{~km}$. The conductivity inside the anomaly and outside it is the same $\sigma_{e}=\sigma_{i}=10^{-2}(\mathrm{ohm} \mathrm{m})^{-1}$. The stress gradient $\Delta P$ is $3 \mathrm{MPa}$. Let us suppose that $\mathrm{AE}$ induces increase $\Delta C$ by $10 \%$ as compared with background value $C=100 \mathrm{mV} / \mathrm{atm}$. Neglecting geometrical factors, $\overline{\mathrm{E}} \simeq 1, \overline{\mathrm{B}} \simeq 1$, the estimates (Eq.16) show that magnitude of electric field disturbance is to be $E \simeq 3 \mu \mathrm{V} / \mathrm{m}$. At the same time the estimated magnitude of magnetic 


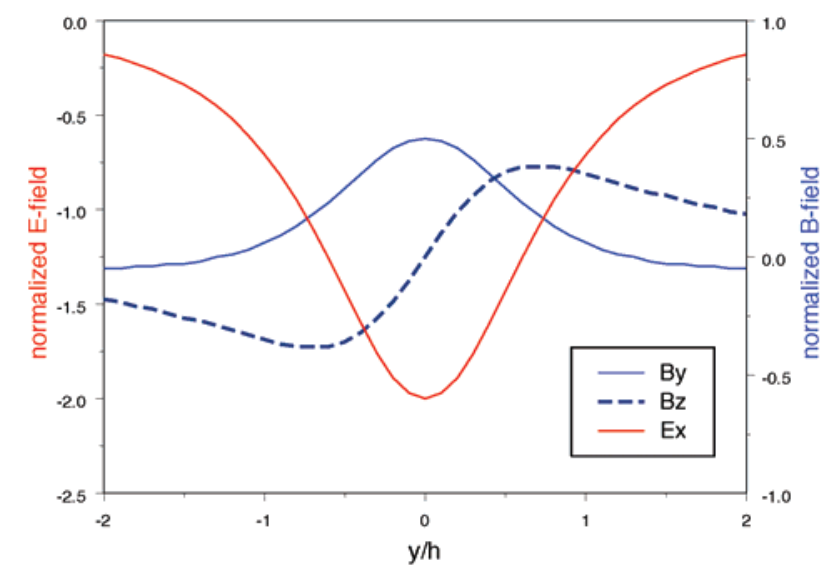

Figure 3. Electromagnetic anomaly above the region with enhanced acoustic emission: the profile across the $\nabla \mathbf{P}$ at $x=0$.
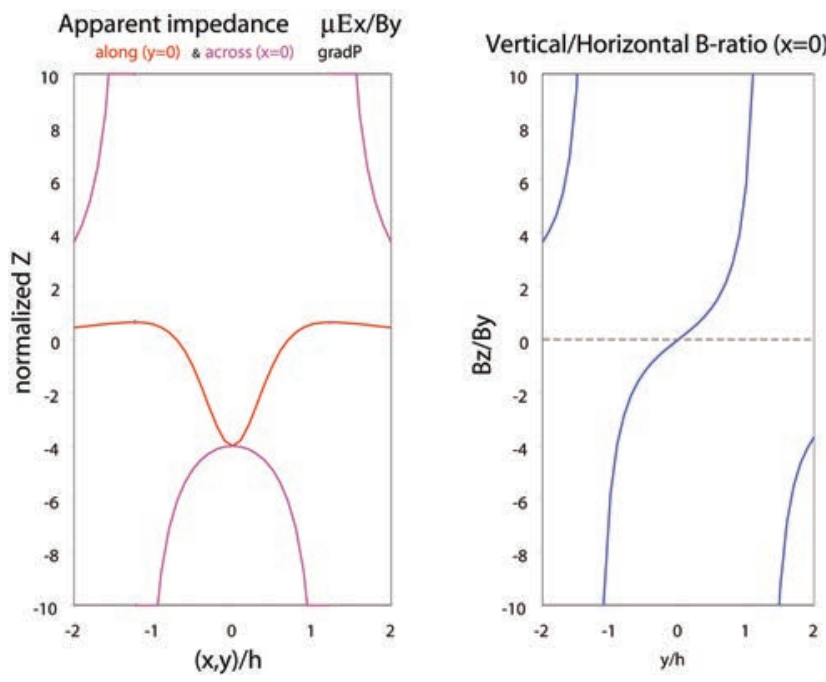

Figure 4. Apparent impedance $E_{x} / H_{y}$ (left panel) along $(\mathrm{y}=0)$ and across $(\mathrm{x}=0) \nabla \mathrm{P}$, and $B_{z} / B_{y}$ value (right panel) of anomalous electromagnetic field. field disturbance is to be $\sim 0.3$ nT. The ULF anomalies of such magnitude can be easily detected by modern search-coil magnetometers in the frequency range $f>10^{-2} \mathrm{~Hz}^{26}$

\section{Discussion}

There were numerous efforts to detect either in laboratory or in field observations simultaneous acoustic and electromagnetic impulses prior to sample destruction or EQ. ${ }^{12,27,28}$ Here we have proposed the mechanism which couples phenomena that differ by typical frequency by 3-4 orders: AE in ELF band, and electromagnetic noise in ULF band. The expected electromagnetic anomalies may manifest themselves in two possible forms. In the case of laminar crust fluid flow, a direct current (DC) telluric and magnetic anomalies above a region with enhanced $\mathrm{AE}$ are to be observed. Realistically, the fluid flow has an irregular character. For example, according to Byerlee $^{29} \mathrm{a}$ fault zone is filled with sealed overpressured compartments, which become connected prior to EQ. In this case, the electromagnetic effect on the ground would be revealed as an occurrence of a region with elevated level of ULF electromagnetic noise.

Observations of $\mathrm{AE}$ in narrow bands 30,160 , $500,1000 \mathrm{~Hz}$ a tunnel inside a rocky mountain at depth of $\sim 100 \mathrm{~m}$ at Matsushiro Seimological Observatory (Japan) were conducted by Gorbatikov et al.. ${ }^{30}$ They observed a definite increase of AE activity starting at about half a day even before weak EQ $(3<M<5.2)$, which decreased after EQ also in about half a day. The characteristic radius of the AE sensitivity was $\sim 120-150 \mathrm{~km}$ for $M \sim 5$ events and expected to be $\sim 400 \mathrm{~km}$ for $M \sim 6$. For the clearest case with $\mathrm{AE}$ enhancement the increase $\sim 2$ times of the integrated magnetic variations in the band $0.01-0.03 \mathrm{~Hz}$ was detected. These observations, showing that before EQs an enhanced $\sim 1 \mathrm{kHz}$ $\mathrm{AE}$ was accompanied by an increased level of $\sim 10 \mathrm{mHz}$ electromagnetic noise, fit the proposed mechanism.

Observations of hydrogeodynamic precursors revealed zones with an abnormally high sensitivity to the preparation stage of EQs, which might be related to $\mathrm{AE}$ induced changes of the filtration parameters. Observations showed that sometimes seismic-related $\mathrm{AE}$ are observed far from the epicenter of impending EQ. Distant generation of $\mathrm{AE}$ is probably caused by local fracturing processes in a distant zone. According to the suggested mechanism the increase of $\mathrm{AE}$ intensity may be accompanied by telluric anomalies. This way the suggested mechanism can explain the distant manifestation of telluric anomalies.

\section{Conclusions}

The evident weak point of the current status of seismo-electromagnetic studies is the lack of cooperation and integration between different types of instruments to measure various terrestrial fields. We have presented a possible example of physical phenomenon which may be revealed only with the help of such coordination. We put forward a hypothesis that $\mathrm{AE}$ caused by a seismic process can modify the streaming potential of underground fluid. The occurrence in the crust of a region with distinct hydrodynamic and electrokinetic parameters (even as low as 10\%) will result in an appearance of anomalous telluric and magnetic fields on the surface above. The estimates with a simple model with an elliptic-shaped inhomogeneity show that the expected electromagnetic signals of electrokinetic origin from AE modified source in the DC-ULF range could reach the detection level. The suggested hypothesis about possible coupling between $\mathrm{AE}$ and geoelectrical anomalies needs observational verification. Verification of this hypothesis requires simultaneous observations of $\mathrm{AE}$ intensity in ELF band, telluric electric and magnetic fields in DC/ULF/ELF bands.

\section{References}

1 Eberhart-Phillips D, Stanley WD, Rodriguez BD, Lutter WJ. Surface seismic and electrical methods to detect fluids related to faulting. J Geophys Res 1995;100:12919-36.

2. Hayakawa M, Fujinawa Y, eds. Electromagnetic phenomena related to earthquake prediction. Tokyo: Terra Scientific; 1994.

3. Hayakawa M, ed. Atmospheric and ionospheric electromagnetic phenomena associated with earthquakes. Tokyo: Terra Scientific; 1999.

4. Hayakawa M, Molchanov 0, eds. Seismo electromagnetics. Tokyo: TERRAPUB; 2002. 
5. Hayakawa M, ed. Earthquake prediction studies: seismo electromagnetics. Tokyo: TERRAPUB; 2013.

6. Kissin IG. Middle- and short-term precursors of earthquakes and their factors determining reliability. J Earthquake Predict Res 1997;6:367-86.

7. Ishido T, Mizutani H. Experimental and theoretical basis of electrokinetic phenomena in rock-water systems and its applications to geophysics. J Geophys Res 1981;86:1763.

8. Mizutani H, Ishido T, Yokokura T, Ohnishi S. Electrokinetic phenomena associated with earthquakes. Geophys Res Lett 1976;3:365-8.

9. Zlotnicki J, Mouel J-L. Possible electrokinetic origin of large magnetic variations at La Fournaise volcano. Nature 1990; 343:633-5.

10. Hattori K. ULF geomagnetic changes associated with large earthquakes. Terrestr Atmos Ocean Sci 2004;15:329-60.

11. Makarenko VN, Groza AA. Earthquake precursors and acoustic fields. Geophys J 1991;13:3-13.

12. Yamada I, Masuda K, Mizutani $H$. Electromagnetic and acoustic emission associated with rock fracture. Phys Earth Planet Inter 1989;57:157-68.

13. Marcak H. Acoustic emission from rock under stress and hydraulic pressure. J Appl Geophys 1994;31:205-12.

14. Smirnov VB, Ponomarev AV, Zavyalov AD. Acoustic vibration structure in rock samples and seismic process. Fizika Zemli (Solid Earth Physics) 1995;1:38-58.
15. Diakonov BP, Karyev BS, Khavroshkin OB, et al. Manifestation of Earth deformation process by high frequency seismic noise characteristics. Phys Earth Planet Inter 1990;63:151-62.

16. Morgunov VA, Lubashevsky MN, Fubrizius VZ, Fubrizius ZE. Geoacoustic precursor of Spitak earthquake. Volcanol Seismol 1991;4:104-7.

17. Chersky NV, Zarev VP, Konovalova VM, Kuznezov OL. Influence of ultrasound fields on the crust's permeability under water filtration. Doklady Akademii Nauk SSSR 1977;232:201-5.

18. Zarev VP, Kuznezov OL. Experimental study of physical and chemical processes in the rocks under moderate deformations, Fizika Zemli (Solid Earth Physics) 1978;6:94-101.

19. Jouniaux L, Ishido T. Electrokinetics in Earth sciences: a tutorial. Int J Geophys 2012;2012:286107.

20. Fitterman DV. Electrokinetic and magnetic anomalies associated with dilatant regions in a layered earth. J Geophys Res 1978;83:5923.

21. Fenoglio MA, Johnston MJS, Byerlee JD. Magnetic and electric fields associated with changes in high pore pressure in fault zones - application to the LomaPrieta ULF emissions. J Geophys Res 1995;100:12951-8.

22. Fedorov E, Pilipenko V, Uyeda S. Electric and magnetic fields generated by electrokinetic processes in a conductive crust. Phys Chem Earth 2001;26:793-9.

23. Landau LD, Lifshitz EM. Electrodynamics of continuous media. Reading, MA: Addison-Wesley; 1960.

24. Hayakawa M, Kawate R, Molchanov OA, Yumoto K. Results of ULF magnetic field measurements during the Guam earthquake of 8 August 1993. Geophys Res Lett 1996;23:241-4.

25. Pilipenko V, Nenovski P, Tanaka H. Detection and discrimination of VLF/ULF seismic-related electromagnetic emissions. Bulgarian Geophys J 2005;29:13-30.

26. Shiokawa K, Nomura R, Sakaguchi K, et al. The STEL induction magnetometer network to observe high-frequency geomagnetic pulsations. Earth Planet Space 2010;62:517-24.

27. Lifshitz VYa, Semertan AA, Fomichov AT. Acoustic and electromagnetic emission during single-axis compression. Doklady AN SSSR 1980;255:821-9.

28. Diakonov VP, Chukhlomin VI, Fadeev VA, et al. Electromagnetic radiation and seismoacoustic emission of rocks in the natural occurrence. Doklady Akademii Nauk SSSR 1986;290:828-9.

29. Byerlee J. Model for episodic flow of highpressure water in fault zones before earthquakes. Geology 1993;21:303-6.

30. Gorbatikov AV, Molchanov OA, Hayakawa $\mathrm{M}$, et al. Acoustic emission possibly related to earthquakes, observed at Matsushiro, Japan, and its implications. In: Hayakawa M, Molchanov OA, eds. Seismo-electromagnetics: lithosphere - atmosphere - ionosphere coupling. Tokyo: TERRAPUB; 2002. pp 1-10. 